\title{
The analysis of $\mathrm{Li}$ I 6708A line through the rotational period of $\mathrm{HD} 166473$ taking into account Paschen-Back magnetic splitting
}

\author{
A. V. Shavrina ${ }^{1}$, V. Khalack ${ }^{2}$, Y. Glagolevskij ${ }^{3}$, D. Lyashko ${ }^{4}$, \\ J. Landstreet ${ }^{5,6}$, F. Leone $^{7}$ and M. Giarrusso ${ }^{7}$ \\ ${ }^{1}$ Main Astronomical Observatory, Kyiv, Ukraine, email: shavrina@mao.kiev.ua \\ ${ }^{2}$ Université de Moncton, Moncton, N.-B., Canada, email: khalakv@umoncton.ca \\ ${ }^{3}$ Special Astrophysical Observatory, Nizhnij Arkhyz, Russia \\ ${ }^{4}$ Tavrian National University, Simferopol, Ukraine \\ ${ }^{5}$ University of Western Ontario, London, Canada \\ ${ }^{6}$ Armagh Observatory, Armagh, Northern Ireland - United Kingdom \\ ${ }^{7}$ Università di Catania, Catania, Italy
}

\begin{abstract}
The analysis of Li I $6708 \AA$ line was performed for 6 rotational phases distributed over the whole rotational period ( $\sim 9.5$ years) of HD166473. The magnetic field model was constructed based on the polarimetric measurements from Mathys et al. (2007). For each observed phase the modulus of the magnetic field was also estimated from simulation of the Fe II $6147 \AA$, $6149 \AA$ and Pr III $6706.7 \AA$ line profiles taking into account Zeeman magnetic splitting. The lithium abundance in each phase was obtained from fitting the observed Li I $6708 \AA$ profile with the synthetic one calculated assuming Paschen-Back splitting and estimated magnetic field characteristics from Pr III $6706.7 \AA$ line profile.
\end{abstract}

Keywords. Stars: chemically peculiar, stars: individual: HD166473

\section{Introduction}

High-resolution spectra of the strongly magnetic roAp star HD166473 taken for eight rotational phases spread from 0.09 to $0.97\left(P_{\text {rot }}=3513^{d} .64\right)$ were analyzed to study the variability of the Li I $6708 \AA$ line profile. The line profiles were analyzed by the method of synthetic spectra using a Kurucz model stellar atmosphere with $T_{\text {eff }}=7750 \mathrm{~K}$ and $\log g=4.0$ (Shavrina et al. 2006). The magnetic splitting of Fe II $6147 \AA, 6149 \AA$ and Pr III $6706 \AA$ lines has been calculated taking into account the Zeeman effect, while for Li I $6708 \AA$ line we have employed the Paschen-Back effect (Khalack \& Landstreet 2012, Stift et al. 2008). The magnetic field measurements of Mathys et al. (2007) have been used to reconstruct a magnetic field configuration employing the method described by Gerth \& Glagolevskij (2003). This reconstruction results in the inclination angle of rotational axis to the line of sight $i=15^{\circ}$ and the angle $\beta=75^{\circ}$.

\section{Abundance analysis}

The abundances of $\mathrm{Li}$ and $\mathrm{Pr}$ obtained from the best fit of observed line profiles are shown in the Table 1. Magnetic splitting of Li I line due to Paschen-Back effect is calculated using magnetic field parameters obtained from the modeling of the Pr III line profile. In this procedure we have employed an idea that Li and REE lines are formed near the magnetic poles (see Shavrina et al. 2001). We have also estimated the abundances of Ce II, Nd II and Sm II whose lines contribute to the Li blend (see Table 1). 
Table 1. Abundance of chemical species at different rotational phases of HD166473.

\begin{tabular}{|c|c|c|c|c|c|c|c|c|c|}
\hline |Phase & $\mid 0.095$ & 0.26 & 0.39 & 0.58 & 0.64 & 0.69 & 0.94 & $0.00^{1}$ & solar $^{2}$ \\
\hline $\begin{array}{l}\log \left(N_{L i I} / N_{H}\right) \\
{ }^{6} L i /{ }^{7} L i \\
\log \left(N_{C \text { eII }} / N_{H}\right) \\
\log \left(N_{P r I I} / N_{H}\right) \\
\log \left(N_{N \text { dII }} / N_{H}\right) \\
\log \left(N_{S \text { III }} / N_{H}\right) \\
\log \left(N_{\text {FeII }} / N_{H}\right)\end{array}$ & $\begin{array}{l}-8.20 \\
0.0 \\
-7.78 \\
-7.76 \\
-8.00 \\
-8.45 \\
-4.37\end{array}$ & -4.42 & \begin{tabular}{|l|}
-8.42 \\
0.5 \\
-7.73 \\
-7.86 \\
-8.10 \\
-8.05 \\
-4.45
\end{tabular} & \begin{tabular}{|l|}
-8.23 \\
0.5 \\
-7.60 \\
-7.82 \\
-8.22 \\
-7.68 \\
-4.35
\end{tabular} & \begin{tabular}{|l|}
-8.20 \\
0.5 \\
-7.63 \\
-7.80 \\
-8.10 \\
-7.68 \\
-4.35
\end{tabular} & \begin{tabular}{|l|}
-8.24 \\
0.5 \\
-7.64 \\
-7.80 \\
-8.10 \\
-7.75 \\
-4.30
\end{tabular} & \begin{tabular}{|l|}
-8.23 \\
0.0 \\
-7.78 \\
-7.76 \\
-8.30 \\
-8.65 \\
-4.45
\end{tabular} & $\begin{array}{l}-7.55 \\
-7.60 \\
-7.97 \\
-8.25 \\
-4.31\end{array}$ & \begin{tabular}{|c|}
-10.95 \\
0.03 \\
-10.42 \\
-11.28 \\
-10.58 \\
-11.04 \\
-4.50
\end{tabular} \\
\hline
\end{tabular}

Notes:

${ }^{1}$ Results of Gelbmann et al. (2000), ${ }^{2}$ solar data are taken from Grevesse et al. (2010)

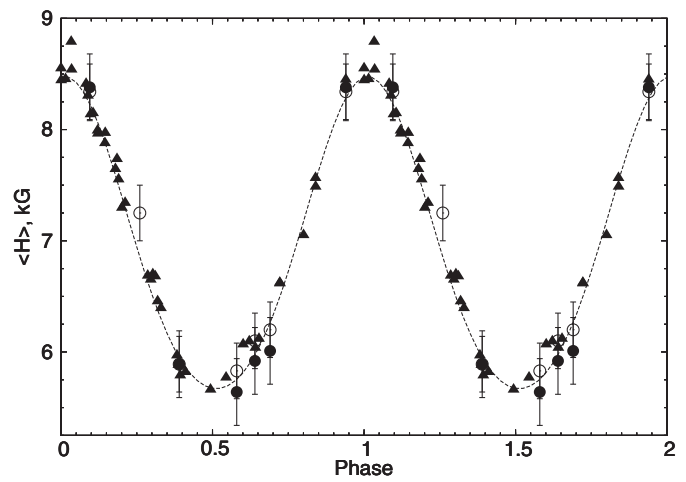

Figure 1. Variation of the mean magnetic field modulus (triangles) with rotational phase for HD166473 (Mathys et al. (2007)) and its approximation (dashed curve) in the framework of Gerth \& Glagolevskij (2003) model. The magnetic field intensity estimated from the analysis of Pr III $6706 \AA$ line (filled circles) and Fe II $6147 \AA$, $6149 \AA$ lines (open circles) accords rather well with the Mathys' data almost for all phases.

One can note that:

- all phases show higher than "cosmic" (-8.7 dex) abundance of lithium;

- some differences in the abundance of $\mathrm{Li}$ I, the isotopic ratio ${ }^{6} \mathrm{Li} /{ }^{7} \mathrm{Li}$, and REE abundances for different phases, and rather different values of the magnetic field strength obtained from the Pr III $6706.7 \AA$ profile, can be explained by the different location of lithium and REE spots, and by their different stratification with optical depth;

- the value of ${ }^{6} \mathrm{Li} /{ }^{7} \mathrm{Li}$ ratio can be consider as a parameter(measure) of model Li line profile disagreement with observed one;

- additional spectra of this very interesting star (high resolution and high $\mathrm{S} / \mathrm{N}$ ) are required to thoroughly cover the whole rotational period.

\section{References}

Gelbmann, M., Ryabchikova, T., Weiss, W. W., Piskunov, N., Kupka, F., \& Mathys, G. 2000, $A \mathscr{E} A, 356,200$

Gerth, E. \& Glagolevskij, Y. 2003, Bulletin of SAO, 56, 25

Grevesse, N., Asplund, M., Sauval, A. J., \& Scott, P. 2010, ApESSS, 328, 179

Khalack, V. \& Landstreet, J. 2012, MNRAS, 427, 569

Mathys, G., Kurtz, D. W., \& Elkin, V. G. 2007, MNRAS, 380, 181

Shavrina, A. V., Polosukhina, N. S., Khan, S., et al. 2006, Astron. Rep., 50, N6, 500

Shavrina, A. V., Polosukhina, N. S., Zverko, J., Khalack, V., Tsymbal, V. V., \& Žižňovský, J. 2001, A\&A 372,571

Stift, M. J., Leone, F., \& Landi Degl'Innocenti, E. 2008, MNRAS 385, 1813 